\title{
Electronic structure and properties of alternating donor-acceptor conjugated copolymers: 3,4-Ethylenedioxythiophene (EDOT) copolymers and model compounds
}

\author{
Chia-Ling Pai ${ }^{a}$, Cheng-Liang Liu ${ }^{a}$, Wen-Chang Chen ${ }^{a, b, *}$, Samson A. Jenekhe ${ }^{c}$ \\ ${ }^{a}$ Department of Chemical Engineering, National Taiwan University, Taipei 106, Taiwan, ROC \\ ${ }^{\mathrm{b}}$ Institute of Polymer Science and Engineering, National Taiwan University, Taipei 106, Taiwan, ROC \\ ${ }^{\mathrm{c}}$ Department of Chemical Engineering and Department of Chemistry, University of Washington, Seattle, WA 98195-1750, USA
}

Received 28 September 2005; received in revised form 24 November 2005; accepted 28 November 2005

Available online 20 December 2005

\begin{abstract}
The electronic structure and properties of 3,4-ethylenedioxythiophene (EDOT) based alternating donor-acceptor conjugated copolymers and their model compounds were studied by the density functional theory (DFT) at the B3LYP level with 6-31G or 6-31G** basis set. The acceptors investigated include thiazole (Z), thiadiazole (D), thienopyrazine (TP), thienothiadiazole (TD), thiadiazolothienopyrazine (TPD), quinoxaline (BP), benzothiadiazole (BD), pyrazinoquinoxaline (BPP), benzobisthiadiazole (BDD), and thiadiazoloquinoxaline (BDP). The torsional angle, intramolecular charge transfer, bridge bond length, and bond length alternation were analyzed and correlated with the electronic properties. It was found that the geometries of the donor-acceptor materials were significantly affected by the ring size and intramolecular charge transfer. The HOMO level, LUMO level, and band gap of the model compounds were well correlated with the acceptor strength. However, the electronic properties of the copolymers did not vary systematically with the acceptor strength due to the change in geometry from model compound to polymer. The aromatic geometry of EDOT-TP model compound is transformed to quinoid in the corresponding copolymer and results in a small band gap $\left(E_{\mathrm{g}}\right)$ of $0.97 \mathrm{eV}$. Large intramolecular charge transfer and the small bond length alternation in the EDOT-BDP copolymer resulted in an $E_{\mathrm{g}}$ of $0.7 \mathrm{eV}$. Hence, these two polymers could have potential applications for transparent conductors or photovoltaic devices. The small effective masses and large HOMO and LUMO bandwidths of PEDOT-TP and PEDOT-BDP make them potential materials for ambipolar thin film transistors. The theoretical results suggest that both the acceptor strength and the stable geometry contribute significantly to the electronic properties of alternating donor-acceptor conjugated copolymers.
\end{abstract}

(C) 2005 Elsevier Ltd. All rights reserved.

Keywords: 3,4-Ethylenedioxythiophene; Theoretical electronic properties; Donor-acceptor

\section{Introduction}

Conjugated polymer systems with donor-acceptor architecture [1-3], including alternating copolymers, blends, and multilayers, have been widely studied for applications as transparent conductors [1-3], light-emitting diodes [4,5], thin film transistors [6,7], and photovoltaic devices [8,9]. We are particularly interested in the alternating donor-acceptor copolymers since their electronic and optoelectronic properties can be tuned efficiently by intramolecular charge transfer (CT)

\footnotetext{
* Corresponding author. Address: Department of Chemical Engineering, National Taiwan University, No. 1, Sec. 4, Roosevelt Rd, Taipei 106, Taiwan, ROC. Tel.: +8862236 28398; fax: +886223623040.

E-mail address: chenwc@ntu.edu.tw (W.-C. Chen).
}

0032-3861/\$ - see front matter (C) 2005 Elsevier Ltd. All rights reserved. doi:10.1016/j.polymer.2005.11.083
[1-13]. The interaction between the electron donor (D) and acceptor (A) moieties in such an alternating donor-acceptor copolymer can result in the hybridization of the high-lying HOMO energy level of the donor and low-lying energy levels of the acceptor, leading to a relatively small band gap polymer semiconductor with novel electronic structure and ambipolar charge transport properties. The small band gap is of interest for near-infrared light-emitting diodes [4] whereas the ambipolar charge transport feature of such polymers is of interest in developing ambipolar organic thin film transistors [6,7].

Poly(3,4-ethylenedioxythiophene) (PEDOT) and its derivatives are widely studied conjugated polymers due to their relatively low ionization potential, high conductivity when doped, and good stability $[14,15]$. PEDOT homopolymer is widely used as a conducting and hole-injecting electrode in organic light emitting diodes (OLEDs) [4,5]. Several EDOTbased donor-acceptor conjugated polymers with small band 
gaps have been reported, including EDOT-pyridine [16,17], EDOT-(4-dicyanomethylene-4H-cyclopenta[2,1-b;3,4$\left.\mathrm{b}^{\prime}\right]$ dithiophene) $[18,19]$, EDOT-thienopyrazine [20,21], EDOT-benzothiadiazole [22], and EDOT- $\left(N^{\prime} 2^{\prime}\right.$-ethylene-4,5dicarboxylic imide benzothiophene) [23]. The optical or electrochemical band gap of these alternating copolymers were either around $1.0 \mathrm{eV}$ or less and thus transparent conductors or electrochromic applications have been suggested. The electronic properties of these donor-acceptor copolymers varied significantly with the acceptor moiety. A comprehensive understanding of the electronic structures and the electronic properties of these EDOT-based donor-acceptor alternating conjugated polymers would help to further develop this and other classes of donor-acceptor copolymers for electronic applications.

In this paper, we report a theoretical study of the electronic structure and properties of EDOT-based alternating donoracceptor conjugated polymers (4) and their model compounds (2) whose molecular structures are shown in Fig. 1. Ten copolymers and 10 model compounds were investigated, corresponding to ten different electron acceptors. The 10 electron acceptors can be grouped into different backbone ring structure: (a) five-member ring: thiazole (Z), thiadiazole (D), thienopyrazine (TP), thienothiadiazole (TD), thiadiazolothienopyrazine (TPD); and (b) six-member ring: quinoxaline (BP), benzothiadiazole (BD), pyrazinoquinoxaline (BPP), thiadiazoloquinoxaline (BDP), and benzobisthiadiazole (BDD). The model compounds and their corresponding polymers are named as dEDOT (1), EDOT-acceptor (2), PEDOT (3), PEDOT-acceptor (4), respectively. For example, the model compound of 3,4-ethylenedioxythiophene-thienopyrazine and its corresponding polymer are named as EDOT-TP and PEDOT-TP, respectively. The series of ten acceptors provide a basis for a comprehensive understanding of the effects of the backbone ring, heteroatom, and fused rings on the geometric and electronic properties of the model compounds and copolymers. The electronic structure and properties were investigated by the density functional theory (DFT) at the B3LYP level and 6-31G or 6-31G** basis set. The bond length of the EDOT-acceptor bridge, bond length alternation, and intramolecular charge transfer of the materials were analyzed and correlated with their chemical structures. The effects of the acceptor strength on the electronic properties, including the HOMO level, LUMO level, band gap, bandwidth, and effective mass were also studied.

\section{Theoretical analysis}

\subsection{Methodology}

The ground-state geometries and electronic structures of the EDOT-based conjugated polymers and their model compounds were optimized by means of the hybrid density functional theory (DFT) method, using periodic boundary conditions, at the B3LYP level of theory with the 6-31G or 6-31G** basis set performed on Gaussion03 program package [24]. In the case of the model compounds, both basis sets $\left(6-31 \mathrm{G}\right.$ and $\left.6-31 \mathrm{G}^{* *}\right)$ were used in the analysis. The comparison of the theoretical results from the $6-31 \mathrm{G}$ and $6-31 \mathrm{G}^{* *}$ basis sets suggests that both methods give rise to similar theoretical results for

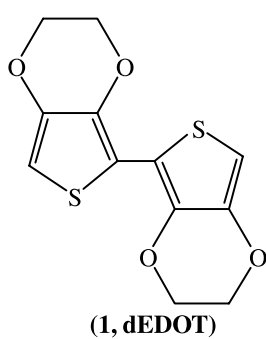

Model Compound

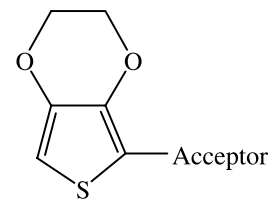

(2, EDOT-Acceptor)

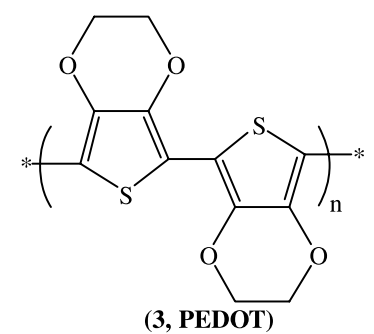

Acceptors
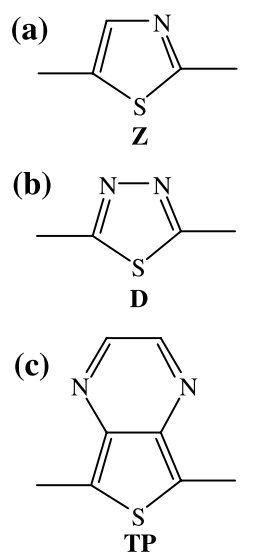

(d)

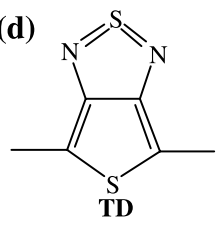

(e)

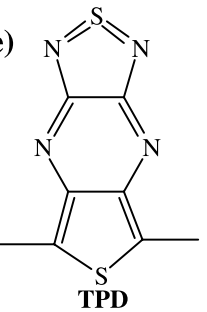

(f)

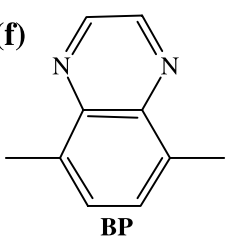

(g)

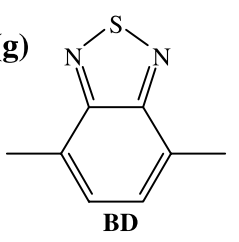

(h)

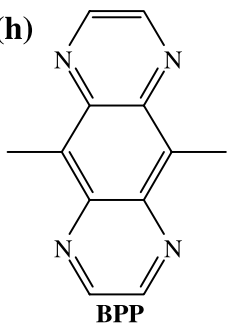

Polymer

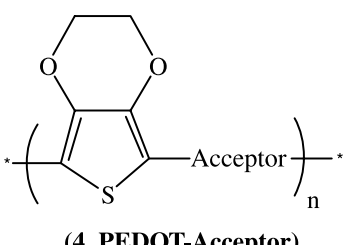

(4, PEDOT-Acceptor) (i)

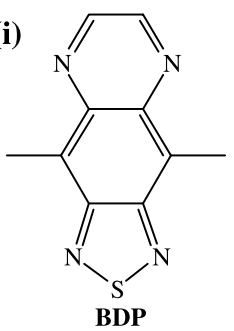

(j)

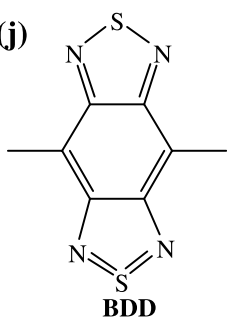

Fig. 1. Molecular structures of EDOT-based alternating donor-acceptor conjugated copolymers and their model compounds. 
the model compounds; we note that the $6-31 \mathrm{G}^{* *}$ basis set considers the orbital polarization whereas the $6-31 \mathrm{G}$ does not. Hence, only the DFT/B3LYP/6-31G was used to analyze the copolymers since the DFT/B3LYP/6-31G** would be timeconsuming. All-trans arrangement was used for the present study since it would be more favorable to the $\pi$-conjugation, lower energy gas phase structure, which was commonly calculated in the thiophene oligomer or polymer systems [25].

\subsection{Geometrical parameters and properties analyzed}

The definitions of torsional angle $(\varphi)$, intramolecular charge transfer $\left(D_{\mathrm{CT}}\right)$, bridge length $\left(L_{\mathrm{B}}\right)$, and bond length alternation of donor $\left(\delta_{\mathrm{D}}\right)$ are shown in Fig. 2 by using compound EDOTBDP as an example. Tosional angle is the deviation from coplanarity between the donor and acceptor, as shown in Fig. 2(a). Bond length alternation (BLA) is the bond length difference between single and double bonds, and represents a major contribution to the existence of a finite band gap in conjugated polymers. Since there is no obvious definition of BLA for the complicated structures of the model compounds, it might be feasible to just investigate the BLA of the invariable EDOT donor, which is defined as $\delta_{\mathrm{D}}$. By using compound EDOT-BDP as an example, the $\delta_{\mathrm{D}}$ is calculated as the difference between the bond length of $\mathrm{C}_{2}-\mathrm{C}_{2}(1.438 \AA)$ and the average bond length of $\mathrm{C}_{1}-\mathrm{C}_{2}(1.361 \AA)$ and $\mathrm{C}_{2}-\mathrm{C}_{3}(1.377 \AA)$, as shown in Fig. 2(a). The $L_{\mathrm{B}}$ is the bond length between the donor and acceptor, as shown in the $\mathrm{C}_{3}-\mathrm{C}_{4}$ of Fig. 2(a). Intramolecular charge transfer was calculated as the average of the summation of Mulliken charge distribution of the donor or acceptor. For example, the $D_{\mathrm{CT}}$ is a summation of all charges for the EDOT ring $(0.287+0.294-0.541-0.535+0.363+$ $0.438-0.321-0.406+0.495=0.073)$, as shown in Fig. 2(b).

Band structures were calculated along the $k$ vector of the one-dimensional copolymers with $30 k$ points in the first Brillouin zone. The energy levels of the HOMO and LUMO were determined from the maximum point of the highest occupied molecular orbital and the minimum point of the lowest unoccupied molecular orbital, respectively. The band gap was then obtained from the minimum difference between the HOMO and LUMO energy levels at a constant $k$. Since the theoretical calculation in this energy level is systematic, the orbit energy can be assumed to verify trend reliably [26,27]. The bandwidth was calculated from the spread of energy levels available to carriers within each band. The effective mass of carriers at the band edge representing mobility was obtained as the square of $\hbar$ multiplied by the reciprocal of the curvature from $E(k)$ with $k$.

\section{Results and discussion}

\subsection{Optimized geometries and electronic properties of model compounds}

The theoretical results from the DFT method can be justified by comparing with the experimental results reported in the literature. To verify the theoretical geometry, the experimental $\mathrm{X}$-ray diffraction data for EDOT-thienopyrazine-EDOT was compared with the theoretical result $[28,29]$, which is similar to compound EDOT-TP. The experimental results showed that the $\mathrm{S}-\mathrm{O}$ and $\mathrm{S}-\mathrm{N}$ intramolecular interaction distances were 2.90 and $2.86 \AA$, respectively. Our theoretical calculation showed that the S-O distance was 2.93 and $2.94 \AA$ from the 6-31G and $6-31 \mathrm{G}^{* *}$ basis sets, respectively, and that of the S-N was $3.00 \AA$ from both $6-31 \mathrm{G}$ and $6-31 \mathrm{G}^{* *}$ basis sets. The comparison suggests that the accuracy of the theoretical geometry of the donor-acceptor model compound in the present study is good. The reported experimental $E_{\mathrm{g}}$ of EDOT$\mathrm{TP}$ is $2.72 \mathrm{eV}$ [20] whereas the calculated values from the 6-31G and 6-31G** basis sets are 2.80 and $2.88 \mathrm{eV}$, respectively. The calculated $E_{\mathrm{g}}$ of EDOT-Z and EDOT-BD as discussed later also shows that the values from $6-31 \mathrm{G}$ are
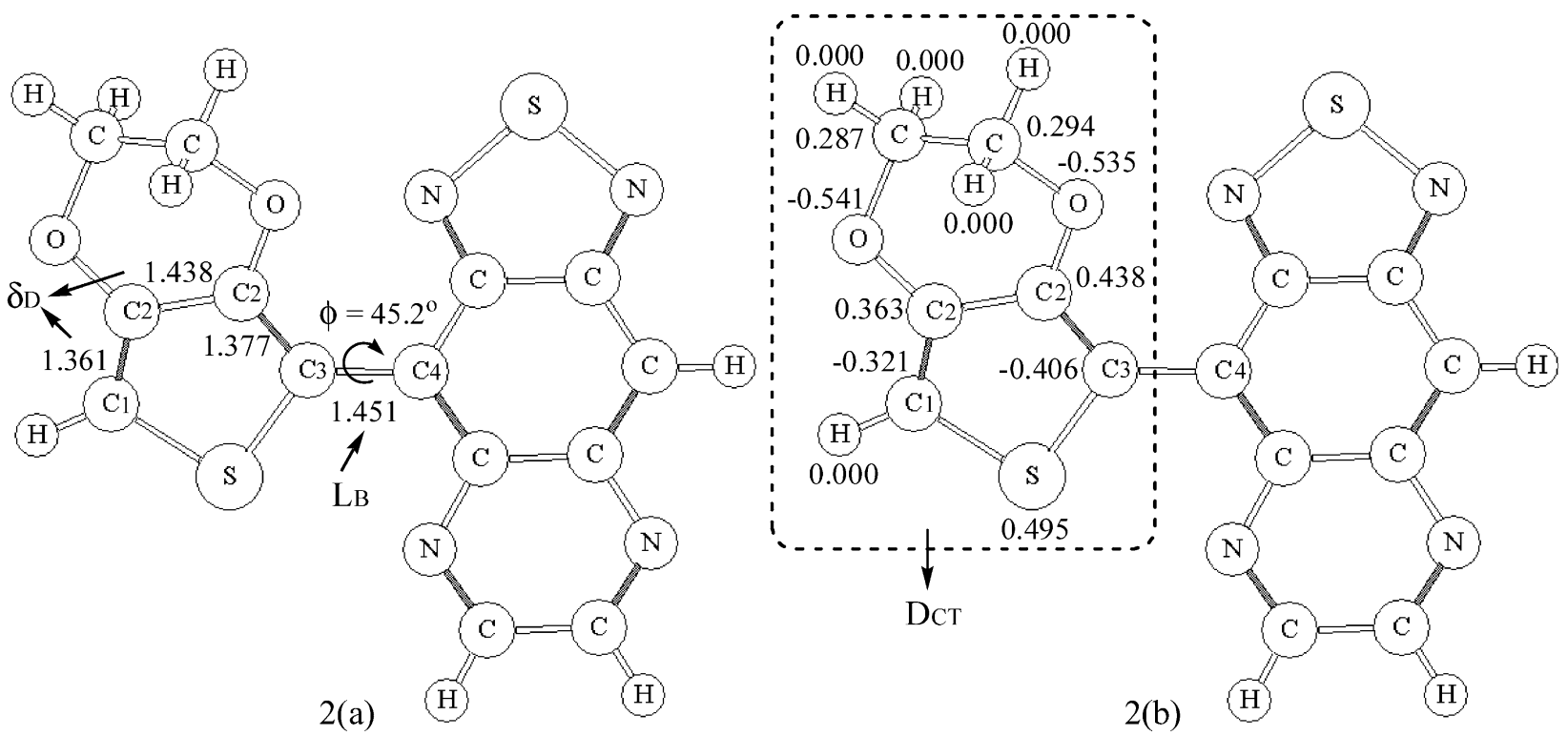

Fig. 2. Optimum geometry (left, unit: $\AA$ ) and Mulliken charge distribution (right, unit: e) of the model compound EDOT-BDP. 
Table 1

The optimum geometry results $\left(\varphi, D_{\mathrm{CT}}, L_{\mathrm{B}}\right.$, and $\left.\delta_{\mathrm{D}}\right)$ for model compounds $\mathbf{1}$ and $\mathbf{2}$

\begin{tabular}{|c|c|c|c|c|c|c|c|c|}
\hline \multirow[t]{2}{*}{ Model compound } & \multicolumn{4}{|c|}{ B3LYP/6-31G } & \multicolumn{4}{|c|}{ B3LYP/6-31G** } \\
\hline & $\varphi\left(^{\circ}\right)$ & $D_{\mathrm{CT}}(\mathrm{e})$ & $L_{\mathrm{B}}(\AA)$ & $\delta_{\mathrm{D}}(\AA)$ & $\varphi\left(^{\circ}\right)$ & $D_{\mathrm{CT}}(\mathrm{e})$ & $L_{\mathrm{B}}(\AA)$ & $\delta_{\mathrm{D}}(\AA)$ \\
\hline DEDOT & 0.1 & 0.000 & 1.429 & 0.070 & 0.1 & 0.000 & 1.443 & 0.058 \\
\hline EDOT-Z & 0.2 & 0.038 & 1.432 & 0.074 & 1.1 & 0.036 & 1.444 & 0.063 \\
\hline EDOT-D & 0.5 & 0.122 & 1.426 & 0.069 & 0.3 & 0.093 & 1.440 & 0.058 \\
\hline EDOT-TP & 0.3 & 0.062 & 1.424 & 0.065 & 0.5 & 0.058 & 1.437 & 0.054 \\
\hline EDOT-TD & 0.7 & 0.088 & 1.414 & 0.059 & 0.7 & 0.070 & 1.432 & 0.053 \\
\hline EDOT-TPD & 0.7 & 0.139 & 1.413 & 0.056 & 0.8 & 0.106 & 1.430 & 0.049 \\
\hline EDOT-BP & 4.9 & -0.030 & 1.460 & 0.068 & 6.8 & -0.036 & 1.465 & 0.054 \\
\hline EDOT-BD & 4.2 & -0.001 & 1.453 & 0.067 & 5.4 & -0.019 & 1.459 & 0.053 \\
\hline EDOT-BPP & 50.8 & 0.043 & 1.458 & 0.075 & 50.5 & 0.009 & 1.465 & 0.061 \\
\hline EDOT-BDP & 45.2 & 0.073 & 1.451 & 0.069 & 44.4 & 0.025 & 1.458 & 0.057 \\
\hline EDOT-BDD & 38.7 & 0.106 & 1.439 & 0.063 & 40.8 & 0.036 & 1.452 & 0.055 \\
\hline
\end{tabular}

closer to the experimental results of similar compounds than those from the 6-31G** $[22,25]$. Hence, comparison of the electronic properties of the model compounds will be based on the results from the 6-31G basis set.

The optimized geometries of model compounds obtained with the 6-31G and 6-31G** basis sets are shown in Supporting Information, respectively. The trend of the geometry of the model compounds obtained from the $6-31 \mathrm{G}$ or $6-31 \mathrm{G}^{* *}$ basis set is similar. The torsional angle $(\varphi)$, intramolecular charge transfer $\left(D_{\mathrm{CT}}\right)$, bridge length $\left(L_{\mathrm{B}}\right)$, and bond length alternation $\left(\delta_{\mathrm{D}}\right)$ are listed in Table 1. As shown in Table 1, the almost zero torsional angle $(\varphi)$ of model compounds (2a-2e) from both DFT/B3LYP/6-31G and 6-31G** level calculations suggests that they have a nearly coplanar conformation. The order on the $D_{\mathrm{CT}}$ obtained from the Mulliken charge distribution with the $6-31 \mathrm{G}$ or $6-31 \mathrm{G} * *$ level is EDOT-TPD $>$ EDOT-D $>$ EDOTTD $>$ EDOT-TP $>$ EDOT-Z. We note that the LUMO levels of the Z, D, TP, TD, and TPD moieties obtained from the 6-31G level are $-1.049,-1.669,-2.518,-3.415$, and $-4.136 \mathrm{eV}$, respectively. The lower the LUMO energy level, the higher the acceptor strength. Hence, the trend of the intramolecular charge transfer is similar to that of the acceptor strength except in the case of EDOT-D. The unusually large $D_{\mathrm{CT}}$ of EDOT-D backbone is probably a result of the two adjacent imine nitrogen atoms with high electronegativity in the thiadiazole ring, which could localize electrons while the adjacent EDOT localize a hole. The order of the $L_{\mathrm{B}}$ between EDOT and the acceptor moiety from either the $6-31 \mathrm{G}$ or $6-31 \mathrm{G}^{* *}$ level is EDOT-TPD $<$ EDOT-TD $<$ EDOT-TP $<$ EDOT-D $<$ EDOT$\mathrm{Z}$, which is in the reverse trend as that of the acceptor strength. It can be explained from the formation of the mesomeric structures induced by intramolecular charge transfer, that is, $\mathrm{D}-\mathrm{A} \leftarrow \rightarrow \mathrm{D}^{+}=\mathrm{A}^{-}$. Furthermore, the intramolecular charge transfer significantly enhances the $\pi$-electron delocalization and thus decreases the BLA of the model compounds. Since the EDOT donor is the same for all the compounds, the donor BLA $\left(\delta_{\mathrm{D}}\right)$ calculated from the $6-31 \mathrm{G}$ or $6-31 \mathrm{G}^{* *}$ level decreases as the acceptor strength increases. These results suggest that the $D_{\mathrm{CT}}, L_{\mathrm{B}}$, and BLA can be controlled by the acceptor strength in the coplanar donor-acceptor model compounds. The trends of $D_{\mathrm{CT}}, L_{\mathrm{B}}$, and BLA obtained from the $6-31 \mathrm{G}$ and $6-31 \mathrm{G}^{* *}$ basis sets are the same for the model compounds although the bond length involved with $\mathrm{S}$ and $\mathrm{O}$ atoms of the former would be elongated.

The large torsional angle $(\varphi)$ of the five model compounds $(\mathbf{2} \mathbf{f}-\mathbf{2} \mathbf{j})$ with six-member ring acceptors suggest that strong steric hindrance exist between the donor and acceptor moieties. The order of the $\varphi$ value obtained from DFT/B3LYP/6-31G or $6-31 \mathrm{G}^{* *}$ is EDOT-BPP $>$ EDOT-BDP $>$ EDOT-BDD $>$ EDOT-BP $>$ EDOT-BD. The large value of $\varphi$ and its variation among these acceptors results from the different size and numbers of fused rings. The above order suggests that the torsional angle of the model compounds with a tricyclic ring is larger than that of a bicyclic one, and that those fused with the pyrazine ring are larger than that fused with the thiadiazole ring. Besides, the model compounds $\mathbf{2 a - 2 e}$ are more planar than $\mathbf{2 f}-\mathbf{2} \mathbf{j}$ due to their additional intramolecular non-covalent interaction between the oxygen donor and the sulfur acceptor. The smaller size of the five-member ring of compounds $\mathbf{2 a - 2 e}$ compared with the six-member ring of $\mathbf{2} \mathbf{f}-\mathbf{2} \mathbf{j}$ also contributes to the smaller $\varphi$ of the former. The order of the intramolecular charge transfer $D_{\mathrm{CT}}$ obtained from the Mulliken charge distribution with the $6-31 \mathrm{G}$ or $6-31 \mathrm{G}^{* *}$ level is EDOTBDD $>$ EDOT-BDP $>$ EDOT-BPP $>$ EDOT-BD $>$ EDOTBP. Note that the LUMO levels of the BP, BD, BPP, BDP, and BDD moieties obtained from the 6-31G level are -2.032 , $-2.802,-2.975,-3.651$, and $-4.316 \mathrm{eV}$, respectively. The trend of the intramolecular charge transfer is exactly the same as that of the acceptor strength. The trend of $L_{\mathrm{B}}$ with the acceptor strength obtained from the $6-31 \mathrm{G}$ or $6-31 \mathrm{G}^{* *}$ level is also similar except EDOT-BD and EDOT-BPP, which are out of order. The order of $\delta_{\mathrm{D}}$ of model compounds $2 \mathbf{2}-\mathbf{2} \mathbf{j}$ shown in Table 1 does not correlate with the acceptor strength or intramolecular charge transfer but is of the same order with the torsional angle. This indicates that single bond character is required for the model compound with a larger $\varphi$ to rotate and thus diminish the double bond character of the mesomeric structures induced by intramolecular charge transfer. The above results also suggest that the $D_{\mathrm{CT}}$ and $L_{\mathrm{B}}$ can be controlled by the acceptor strength in donor-acceptor model compounds. However, the BLA is largely dependent on the $\varphi$ rather than on the acceptor strength.

Fig. 3 shows the HOMO and LUMO energy levels of the model compounds calculated from the 6-31G basis set. 

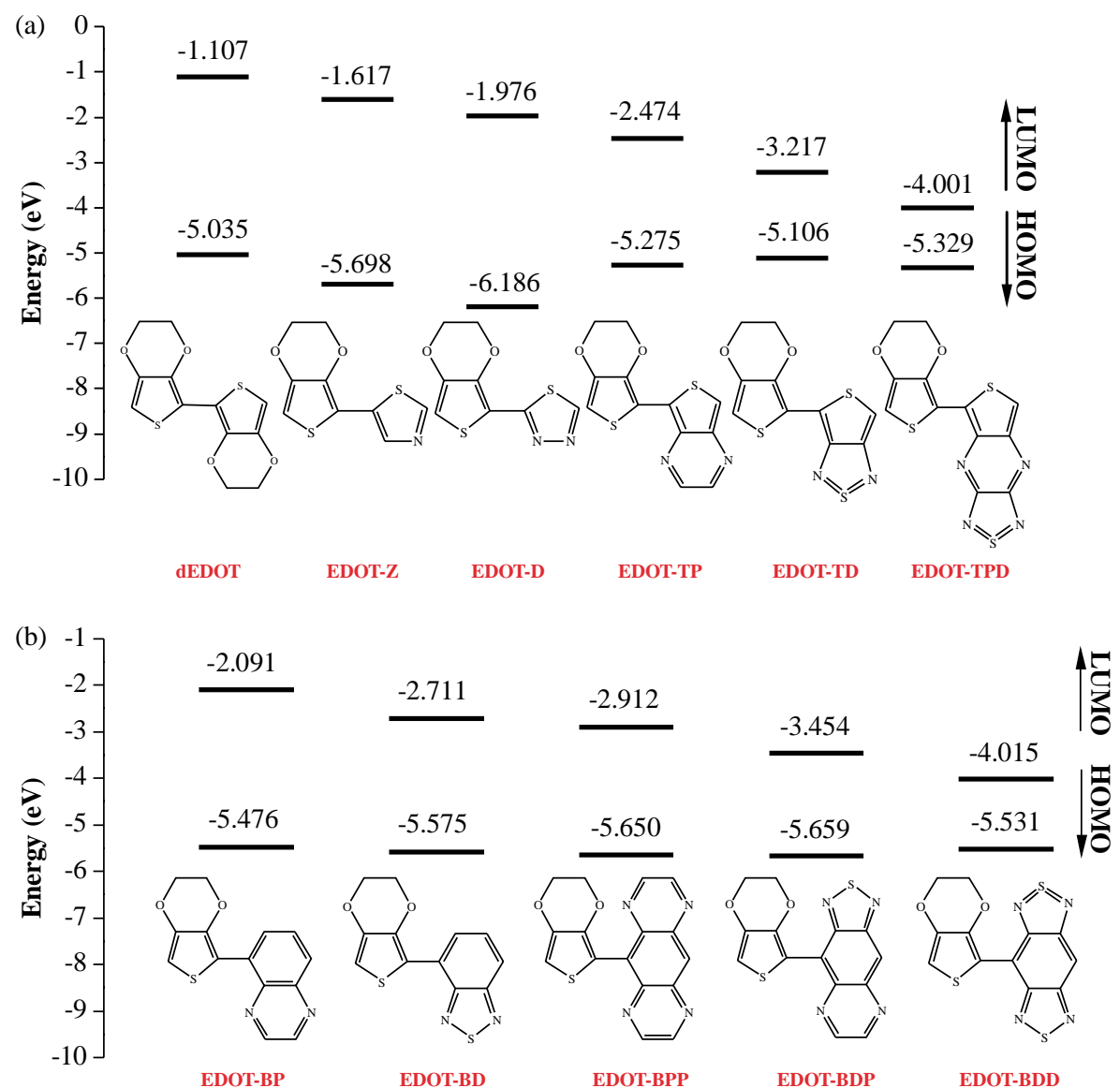

Fig. 3. HOMO and LUMO energy levels of the model compounds by DFT/B3LYP/6-31G basis set (a) acceptors with five-member ring and (b) six-member ring on the backbone (unit: $\mathrm{eV}$ ), respectively.

The calculated HOMO level, LUMO level, and $E_{\mathrm{g}}$ are summarized in Table 2. Although the HOMO/LUMO energy levels and $E_{\mathrm{g}}$ calculated from the $6-31 \mathrm{G}$ and $6-31 \mathrm{G}^{* *}$ basis sets show a similar trend among the ten compounds, those based on the former basis set are smaller. A significant deviation is observed for the LUMO energy level of the model compounds fused with the thiadiazole ring. As shown in the Fig. 3(a), the LUMO energy level of model compounds 1 and $\mathbf{2 a - 2 e}$ decreases in the order: dEDOT $>$ EDOT $-Z>$ EDOT-D $>$ EDOT-TP $>$ EDOT-TD $>$ EDOT-TPD, which is the same order as the acceptor strength. However, the HOMO energy level of these compounds is almost unchanged for compounds dEDOT, EDOT-TP, EDOT-TD, and EDOT-TPD, being -5.035 to $-5.329 \mathrm{eV}$. The much lower $E_{\mathrm{g}}$ of EDOT-TP, EDOT-TD, and EDOT-TPD compared to that of dEDOT indicates a significant effect of intramolecular charge transfer. However, the $E_{\mathrm{g}}$ values of EDOT-Z and EDOT-D are larger than that of dEDOT. In both EDOT-Z and EDOT-D, the lowering of the LUMO level by the presence of the acceptor moiety is more than compensated by the lowering of the

Table 2

The theoretical electronic properties of model compounds $\mathbf{1}$ and $\mathbf{2}$

\begin{tabular}{|c|c|c|c|c|c|c|}
\hline \multirow[t]{2}{*}{ Model compound } & \multicolumn{3}{|c|}{ B3LYP/6-31G } & \multicolumn{3}{|c|}{ B3LYP/6-31G** } \\
\hline & HOMO (eV) & LUMO $(\mathrm{eV})$ & $E_{\mathrm{g}}(\mathrm{eV})$ & HOMO $(\mathrm{eV})$ & LUMO (eV) & $E_{\mathrm{g}}(\mathrm{eV})$ \\
\hline dEDOT & -5.035 & -1.107 & 3.929 & -4.867 & -0.813 & 4.054 \\
\hline EDOT-Z & -5.698 & -1.617 & 4.081 & -5.475 & -1.294 & 4.181 \\
\hline EDOT-D & -6.186 & -1.976 & 4.210 & -5.918 & -1.609 & 4.310 \\
\hline EDOT-TP & -5.275 & -2.474 & 2.800 & -5.114 & -2.238 & 2.876 \\
\hline EDOT-TD & -5.106 & -3.217 & 1.889 & -5.020 & -2.644 & 2.376 \\
\hline EDOT-TPD & -5.329 & -4.001 & 1.328 & -5.170 & -3.515 & 1.654 \\
\hline EDOT-BP & -5.476 & -2.091 & 3.385 & -5.341 & -1.975 & 3.366 \\
\hline EDOT-BD & -5.575 & -2.711 & 2.863 & -5.409 & -2.298 & 3.112 \\
\hline EDOT-BPP & -5.650 & -2.912 & 2.737 & -5.487 & -2.749 & 2.739 \\
\hline EDOT-BDP & -5.659 & -3.454 & 2.204 & -5.474 & -3.037 & 2.436 \\
\hline EDOT-BDD & -5.531 & -4.015 & 1.516 & -5.431 & -3.358 & 2.073 \\
\hline
\end{tabular}


HOMO level. A likely origin of this effect is that the backbone nitrogen atom localizes electrons and breaks the symmetry of the structures, thereby increasing the bond length alternation with consequent widening of the band gap of the two model compounds [30].

The LUMO energy level of model compounds $\mathbf{2} \mathbf{f}-\mathbf{2} \mathbf{j}$ shown in Fig. 3(b) decreases in the order: EDOT-BP $>$ EDOT-BD $>$ EDOT-BPP $>$ EDOT-BDP $>$ EDOT-BDD. This suggests that the higher the acceptor strength, the lower the LUMO level. The HOMO energy level is almost constant in the range of -5.476 to $-5.659 \mathrm{eV}$. The $E_{\mathrm{g}}$ of compounds $\mathbf{2 \mathbf { f }}-\mathbf{2 \mathbf { j }}$ is in the range of $1.516-3.385 \mathrm{eV}$, which is significantly lower than that of the parent dEDOT. This reduction of the band gap shows that intramolecular charge transfer is a dominant effect in these donor-acceptor compounds. The correlation between the $E_{\mathrm{g}}$ and the chemical structures of compounds $2 \mathbf{f}-\mathbf{2} \mathbf{j}$ also suggest that the thiadiazole ring is more effective in lowering $E_{\mathrm{g}}$ than the pyrazine ring. The theoretical electronic properties of EDOT-benzene (LUMO, HOMO and $E_{\mathrm{g}}$ ) were calculated to be $-1.044,-5.470$, and $4.426 \mathrm{eV}$, respectively. By comparing the LUMO of the EDOT-benzene with those of compounds $\mathbf{2 f}-\mathbf{2 j}$, we find that the LUMO is lowered by about 1 and $1.5 \mathrm{eV}$ as the benzene ring is fused with the pyrazine and thiadiazole rings, respectively. Besides the consideration of the acceptor strength, those containing the lower LUMO energies of the model compounds containing thiadiazole ring than the pyrazine ring is partly due to larger torsional angle in the later.

\subsection{Optimized geometries and electronic properties of the $D-A$ conjugated copolymers}

Table 3 lists the calculated geometrical and intramolecular charge transfer parameters of the alternating donor-acceptor conjugated copolymers at the DFT/B3LYP/6-31G level. Optimized structures of the polymers are presented in Supporting Information. The almost zero torsional angles of the copolymers $(\mathbf{4 a}-\mathbf{4 e})$ with a five-member ring acceptor suggest nearly a coplanar conformation, which is similar to the results of the model compounds. The order of the intramolecular charge transfer parameter $\left(D_{\mathrm{CT}}\right)$ is PEDOT-D $>$ PEDOT-TPD $>$ PEDOT-TD $>$ PEDOT-Z $>$ PEDOT-TP. This trend is not consistent with the acceptor strength, particularly in the case of PEDOT $-Z$ and PEDOT-D. The deviation is likely

Table 3

The optimum geometry results $\left(\varphi, D_{\mathrm{CT}}, L_{\mathrm{B}}\right.$, and $\left.\delta_{\mathrm{D}}\right)$ of polymers 3 and 4

\begin{tabular}{lrrrl}
\hline Polymer & $\varphi\left({ }^{\circ}\right)$ & $D_{\mathrm{CT}}(\mathrm{e})$ & $L_{\mathrm{B}}(\AA)$ & $\delta_{\mathrm{D}}(\AA)$ \\
\hline PEDOT & 0.3 & 0.000 & 1.420 & 0.039 \\
PEDOT-Z & 0.2 & 0.124 & 1.426 & 0.042 \\
PEDOT,D & 0.2 & 0.237 & 1.427 & 0.042 \\
PEDOT-TP & 0.4 & 0.092 & 1.370 & 0.045 \\
PEDOT-TD & 0.4 & 0.137 & 1.361 & 0.058 \\
PEDOT-TPD & 0.5 & 0.186 & 1.368 & 0.052 \\
PEDOT-BP & 30.4 & -0.018 & 1.456 & 0.042 \\
PEDOT-BD & 30.3 & 0.011 & 1.450 & 0.039 \\
PEDOT-BPP & 47.8 & 0.126 & 1.454 & 0.050 \\
PEDOT-BDP & 38.1 & 0.165 & 1.433 & 0.028 \\
PEDOT-BDD & 25.5 & 0.213 & 1.379 & 0.058 \\
\hline
\end{tabular}

due to the presence of highly electronegative nitrogen atoms in the backbone $\pi$-system in PEDOT-Z and PEDOT-D, as discussed previously. The order on $L_{\mathrm{B}}$ and BLA of the polymers shown in Table 3 is quite different from those of the model compounds. The variation in the geometry between the model compounds and their corresponding polymers might explain the above results. The aromatic geometry of EDOT is the more stable form than the quinoid form in both model compounds and polymers. However, the geometry of the TP moiety shifts from partially aromatic to partially quinoid form when the number of repeating unit increases. As shown in Fig. 4(a), the geometry of EDOT-TP is quite different from that of its corresponding polymer, PEDOT-TP. In EDOT-TP, the partially aromatic form of the model compound is suggested due to the shorter bond lengths of the $\mathrm{C}_{1}-\mathrm{C}_{2}$ $(1.360 \AA), C_{2}-C_{3}(1.379 \AA), C_{4}-C_{5}(1.395 \AA)$, and $C_{5}-C_{6}$ $(1.375 \AA)$ than those of the $\mathrm{C}_{2}-\mathrm{C}_{2}(1.434 \AA), \mathrm{C}_{3}-\mathrm{C}_{4}(1.423 \AA)$, and $\mathrm{C}_{5}-\mathrm{C}_{5}(1.453 \AA)$. However, in the case of PEDOT-TP, the bond lengths of $\mathrm{C}_{1}-\mathrm{C}_{2}(1.422 \AA), \mathrm{C}_{2}-\mathrm{C}_{3}(1.422 \AA), \mathrm{C}_{4}-\mathrm{C}_{5}$ $(1.434 \AA)$, and $\mathrm{C}_{5}-\mathrm{C}_{6}(1.434 \AA)$ are larger than those of the $\mathrm{C}_{2}-$ $\mathrm{C}_{2}(1.377 \AA), \mathrm{C}_{3}-\mathrm{C}_{4}(1.370 \AA)$, and $\mathrm{C}_{5}-\mathrm{C}_{5}(1.424 \AA)$, exhibiting the partially quinoid form. Thus, the geometry of EDOT-TP transforms from an aromatic-dominant to the quinoid-dominant form in PEDOT-TP. The geometry difference between the model compound and its corresponding copolymers is probably due to the resonance of cyclic ring structure and the intramolecular interaction as the size of the unit increases. Fig. 5 shows the possible resonance forms of PEDOT-TP. PEDOT-TP could have four possible resonance forms and probably transfer to the quinoid structure. Although the geometries of PEDOT-TD and PEDOT-TPD are quinoidlike in the thiophene donor, the acceptors are neither quinoidlike nor aromatic-like due to the disruption of $\pi$-conjugation. By using PEDOT-TD of Fig. 4(b) as an example, the bridge bond length of the $\mathrm{C}_{1}-\mathrm{C}_{2}(1.361 \AA)$, and both $\mathrm{C}_{3}-\mathrm{N}(1.321 \AA)$ are double bonds while the bond lengths of the $\mathrm{C}_{2}-\mathrm{C}_{3}$ $(1.439 \AA)$ and $\mathrm{C}_{3}-\mathrm{C}_{4}(1.439 \AA)$ suggest single bonds. Hence, the $\pi$-conjugation along the polymer chain is disrupted by the single bond characteristics of the $\mathrm{C}_{3}-\mathrm{C}_{3}(1.458 \AA)$.

The polymers $\mathbf{4 f}-\mathbf{4 j}$, with acceptors having a six-member ring, have a large torsional angle $(\varphi)$ of $25.5-47.8^{\circ}$, as shown

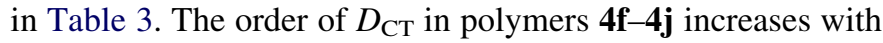
increasing acceptor strength and is the same trend as that of the corresponding model compounds $2 \mathbf{f}-\mathbf{2} \mathbf{j}$. However, in the case of $L_{\mathrm{B}}$ and BLA of polymers $\mathbf{4 f}-\mathbf{4 j}$ (Table 3 ) we could not observe a clear trend and this is quite different from that of model compounds $\mathbf{2 f}-\mathbf{2} \mathbf{j}$. The smallest $\varphi$ observed in PEDOTBDD, among polymers $\mathbf{4} \mathbf{f}-\mathbf{4 j}$ might be due to two factors: (1) The small size of the five-member ring; (2) The double character of the bridge length, as explained below. The geometry of the PEDOT-BDD is shown in Fig. 4(c). The bridge lengths of the $\mathrm{C}_{1}-\mathrm{C}_{2}(1.379 \AA), \mathrm{C}_{3}-\mathrm{N}(1.323,1.325 \AA)$, and $\mathrm{C}_{4}-\mathrm{N}(1.321,1.322 \AA)$ are double bonds while those of the $\mathrm{C}_{2}-\mathrm{C}_{3}(1.463 \AA), \mathrm{C}_{2}-\mathrm{C}_{4}(1.457 \AA), \mathrm{C}_{3}-\mathrm{C}_{5}(1.463 \AA)$, and $\mathrm{C}_{4}-\mathrm{C}_{5}(1.457 \AA)$ are suggested to be single bonds. The $L_{\mathrm{B}}\left(\mathrm{C}_{1}-\right.$ $\mathrm{C}_{2}$ ) of PEDOT-BDD acts as a rigid double bond, similar to those of PEDOT-TD (1.361 ̊) and PEDOT-TPD (1.368 ̊) 
Model Compound

(a)

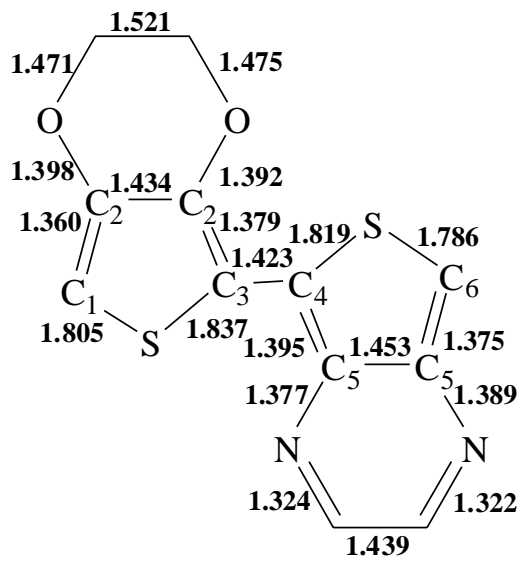

(b)

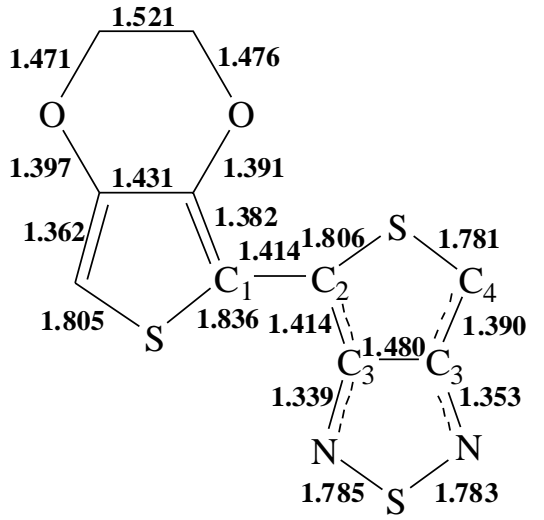

(c)

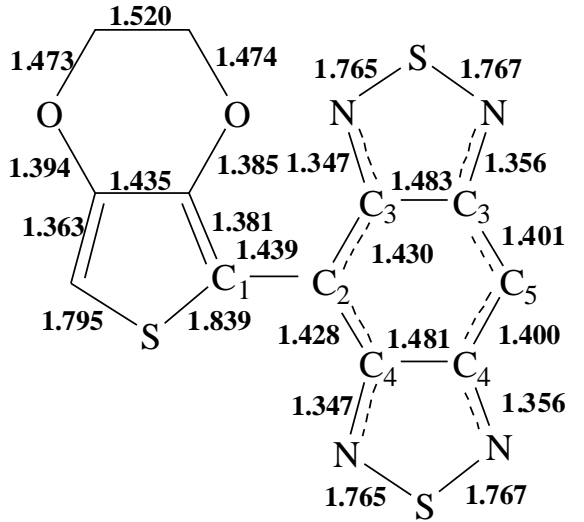

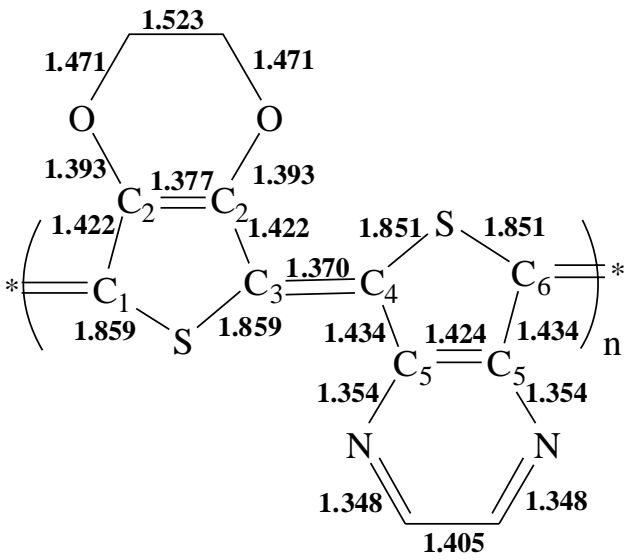

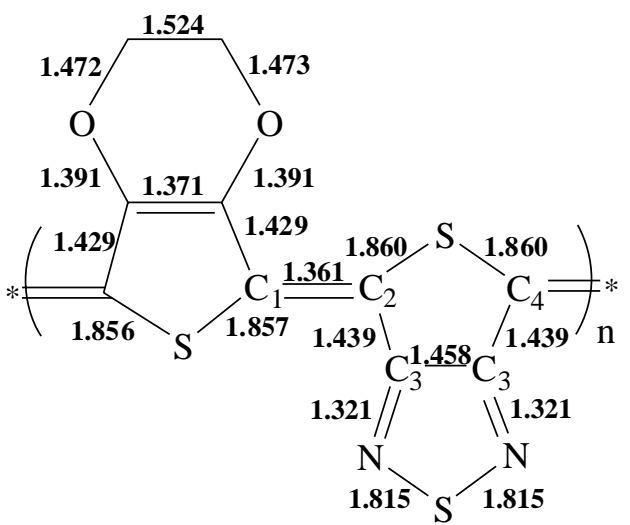

Fig. 4. Optimized structures with the 6-31G basis set of (a) PEDOT-TP, (b) PEDOT-TD, and (c) PEDOT-BDD (unit: $\AA$ ).

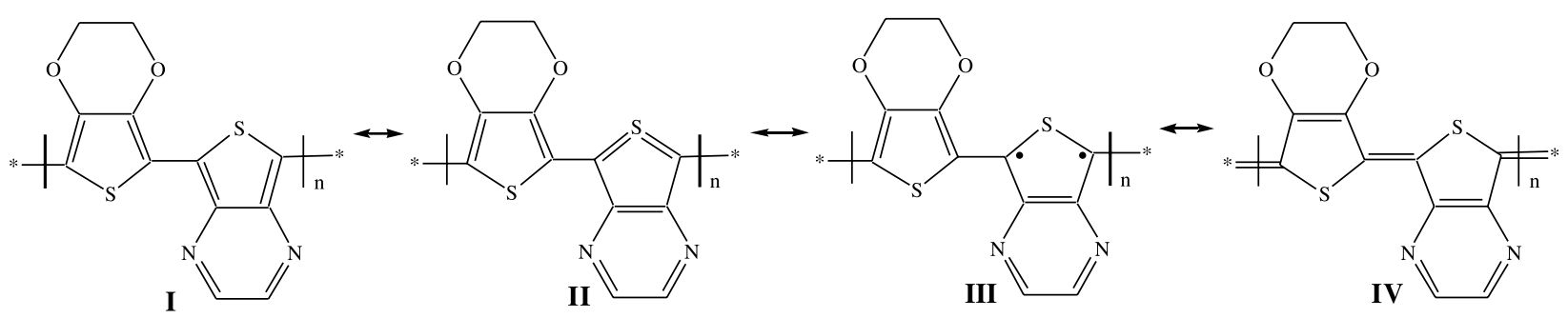

Fig. 5. Resonance form of the PEDOT-TP. 
(Table 3), and contributes partially to the small $\varphi$ of this polymer. However, it is different from the $L_{\mathrm{B}}$ of the other polymers $(\mathbf{4 f}-\mathbf{4 j})$ in the range of $1.433-1.456 \AA$, which is similar to a single bond and thus allowed to rotate and consequently leads to a large $\varphi$. Hence, the $\pi$-conjugation along the polymer chain is disrupted by the single bond characteristics of the $\mathrm{C}_{3}-\mathrm{C}_{3}(1.461 \AA)$ and $\mathrm{C}_{4}-\mathrm{C}_{4}(1.480 \AA)$. Besides, the longer $\mathrm{S}-\mathrm{N}$ bond lengths of the PEDOT-BDD (1.794-1.801 $\AA$ ) than those of its model compound (1.765$1.767 \AA$ ) also suggest that decreased double bond character of the $\mathrm{S}-\mathrm{N}$ bond in the polymer. The same disruption of $\pi$-conjugation that occurs in PEDOT-BDD is also seen in PEDOT-TD and PEDOT-TPD. The same geometrical transformation had also been proposed for poly(thienothiadiazole) and poly(thiophene-thienothiadiazole) reported in the literature [29].

Fig. 6 shows the HOMO and LUMO energy levels of the alternating donor-acceptor conjugated copolymers 4. As shown in Fig. 6(a), the HOMO and LUMO energy levels of polymers $4 \mathbf{a}-4 \mathbf{e}$ do not show a clear trend with that of the acceptor strength. The order of the calculated $E_{\mathrm{g}}(\mathrm{eV})$ is PEDOT-D (2.40) > PEDOT-Z (1.95) > PEDOT-TD (1.79) $>$ PEDOT-TPD (1.69) $>$ PEDOT (1.64) $>$ PEDOT-TP (0.97). The observed different trend of the electronic properties of the polymers compared to those of the model compounds is due primarily to the difference in geometry. The enhancement of the HOMO and LUMO energy levels of PEDOT-TD and
PEDOT-TPD relative to their model compounds is different from the trend of the other three polymers (PEDOT-Z, PEDOT-D, and PEDOT-TP) in which the HOMO is elevated while the LUMO is lowered. The $E_{\mathrm{g}}$ of PEDOT-TD and PEDOT-TPD was similar or higher than those of the corresponding model compounds. The disruption of $\pi$-conjugation of these two polymers might explain this result based on the above geometric analysis [31]. The relatively small band gap of PEDOT-TP is explained as follows. The theoretical $E_{\mathrm{g}}$ of the aromatic and quinoid forms of PEDOT from the DFT/ B3LYP/6-31G theory is 1.886 and $0.254 \mathrm{eV}$, respectively, in which the former is the stable geometry. In the case of poly(thienopyrazine)(P-TP), the theoretical $E_{\mathrm{g}}$ of the stable quinoid form is $1.593 \mathrm{eV}$. As shown in Fig. 4(a), the stable geometry of PEDOT-TP is quinoid and thus explains the origin of the small $E_{\mathrm{g}}$ from the quinoid EDOT moiety. The small $E_{\mathrm{g}}$ characteristic of quinoid poly(thiophene) or poly(ethylenedioxythiophene) has also been reported in the literature [32-34]. Large intramolecular charge transfer could also explain the small $E_{\mathrm{g}}$ of PEDOT-TP among the polymers 4a-4e. Note that PEDOT-TP does not have the problem of localization of charge carrier as seen in PEDOT-Z and PEDOT-D or disruption of $\pi$-conjugation observed in PEDOT-TD and PEDOT-TPD.

As shown in Fig. 6(b), the HOMO and LUMO energy levels of polymers $\mathbf{4 f}-\mathbf{4 j}$ do not show a clear trend with the acceptor strength. PEDOT-BDD has a larger $E_{\mathrm{g}}$ than its model

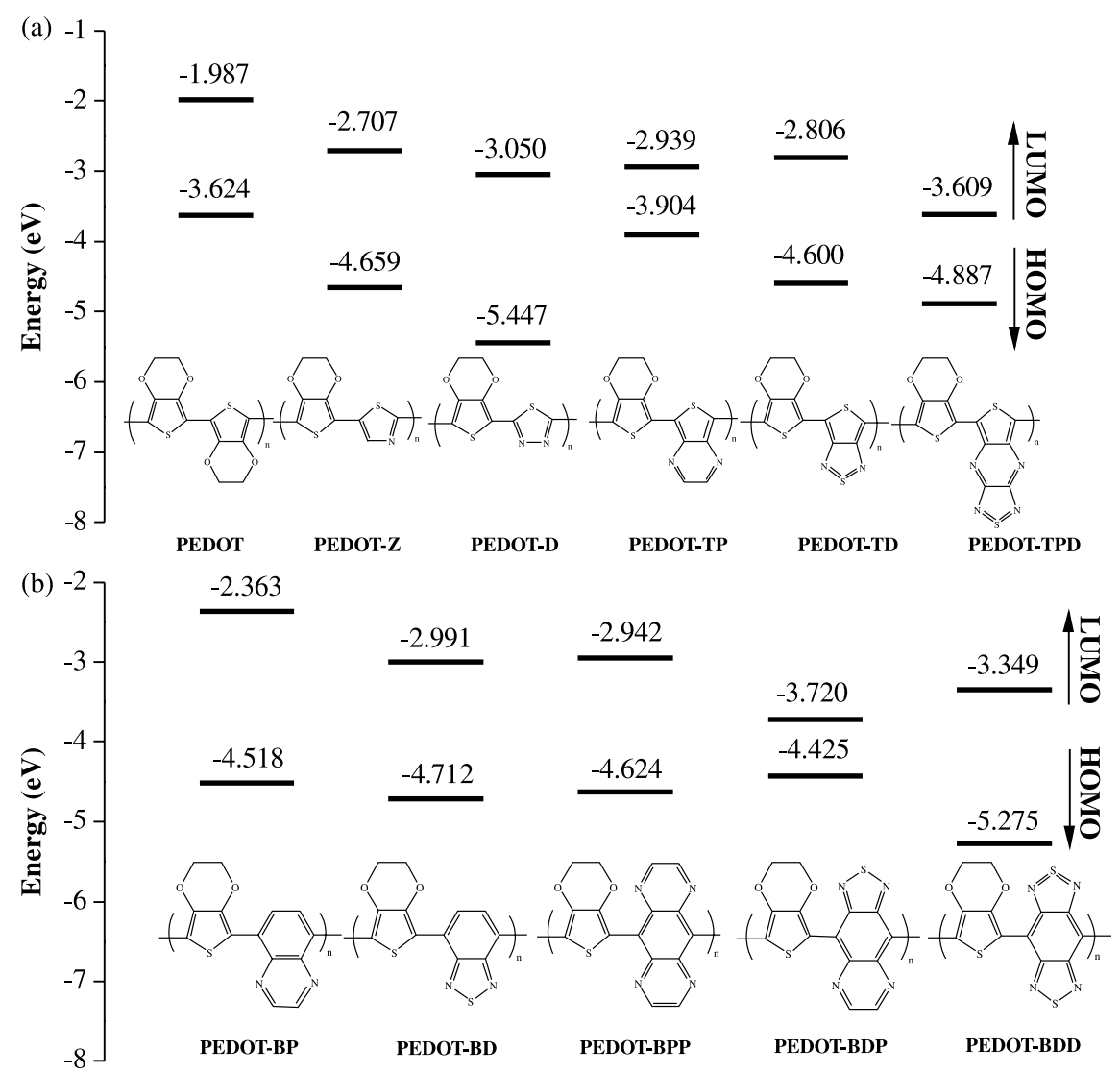

Fig. 6. HOMO and LUMO energy levels by DFT/B3LYP/6-31G basis set (a) acceptors with five-member ring and (b) six-member ring on the backbone (unit: eV), respectively. 
Table 4

The theoretical electronic properties of polymers $\mathbf{3}$ and $\mathbf{4}$

\begin{tabular}{|c|c|c|c|c|c|c|c|}
\hline Polymer & HOMO (eV) & LUMO (eV) & $E_{\mathrm{g}}(\mathrm{eV})$ & Valence BW (eV) & Effective mass, $m_{\mathrm{H}}$ & Conduction BW (eV) & Effective mass, $m_{\mathrm{L}}$ \\
\hline PEDOT & -3.624 & -1.987 & 1.637 & 1.925 & $-0.117 m_{\mathrm{e}}$ & 2.076 & $0.121 m_{\mathrm{e}}$ \\
\hline PEDOT-Z & -4.659 & -2.707 & 1.952 & 1.702 & $-0.143 m_{\mathrm{e}}$ & 1.758 & $0.152 m_{\mathrm{e}}$ \\
\hline PEDOT-D & -5.447 & -3.050 & 2.397 & 1.280 & $-0.189 m_{\mathrm{e}}$ & 1.450 & $0.192 m_{\mathrm{e}}$ \\
\hline PEDOT-TP & -3.904 & -2.939 & 0.965 & 2.022 & $-0.104 m_{\mathrm{e}}$ & 0.938 & $0.130 m_{\mathrm{e}}$ \\
\hline PEDOT-TD & -4.600 & -2.806 & 1.794 & 1.495 & $-0.202 m_{\mathrm{e}}$ & 0.075 & $1.647 m_{\mathrm{e}}$ \\
\hline PEDOT-TPD & -4.887 & -3.609 & 1.686 & 1.289 & $-0.268 m_{\mathrm{e}}$ & 0.491 & $3.311 m_{\mathrm{e}}$ \\
\hline PEDOT-BP & -4.518 & -2.363 & 2.155 & 1.308 & $-0.181 m_{\mathrm{e}}$ & 0.656 & $0.240 m_{\mathrm{e}}$ \\
\hline PEDOT-BD & -4.712 & -2.991 & 1.720 & 1.390 & $-0.160 m_{\mathrm{e}}$ & 0.573 & $0.221 m_{\mathrm{e}}$ \\
\hline PEDOT-BPP & -4.624 & -2.942 & 1.682 & 0.989 & $-0.213 m_{\mathrm{e}}$ & 0.467 & $0.331 m_{\mathrm{e}}$ \\
\hline PEDOT-BDP & -4.425 & -3.720 & 0.704 & 1.473 & $-0.110 m_{\mathrm{e}}$ & 0.729 & $0.146 m_{\mathrm{e}}$ \\
\hline PEDOT-BDD & -5.275 & -3.349 & 1.964 & 0.817 & $-0.329 m_{\mathrm{e}}$ & 0.039 & $7.374 m_{\mathrm{e}}$ \\
\hline
\end{tabular}

compound and this is due to the disruption of $\pi$-conjugation as explained for PEDOT-TD and PEDOT-TPD. Among all polymers 4, the smallest $E_{\mathrm{g}}$ was observed in PEDOT-BDP $(0.70 \mathrm{eV})$. This observation is due to the small donor BLA $(0.028 \AA)$ and the large intramolecular charge transfer $(0.165 \mathrm{e})$ in this copolymer. The small $E_{\mathrm{g}}$ values of PEDOTTP and PEDOT-BDP may have potential applications as transparent conductors ${ }^{1-3}$ or photovoltaic materials ${ }^{8,9}$ since their absorption spectra could extend to the near-infrared region.

The bandwidth (BW) and effective masses of holes and electrons of the polymers are listed in Table 4. Representative band structures of PEDOT-TP and PEDOT-BDP are shown in Fig. 7. The effective mass of holes $\left(m_{\mathrm{H}}\right)$ or electrons $\left(m_{\mathrm{L}}\right)$ does not have a clear correlation with the acceptor strength. PEDOTZ, PEDOT-D, and PEDOT-TP have electron and hole masses that are comparable to those of PEDOT, suggesting good prospects for charge carrier transport. The substitution of nitrogen atoms on the backbone reduces the LUMO energies without significantly impeding carrier mobility through the polymer chain. On the other hand, the large $m_{\mathrm{H}}$ or $m_{\mathrm{L}}$ of PEDOT-TD, PEDOT-TPD, or PEDOT-BDD indicates poor $\pi$-electron delocalization and potential for strong carrier localization. This is as expected since the disruption of
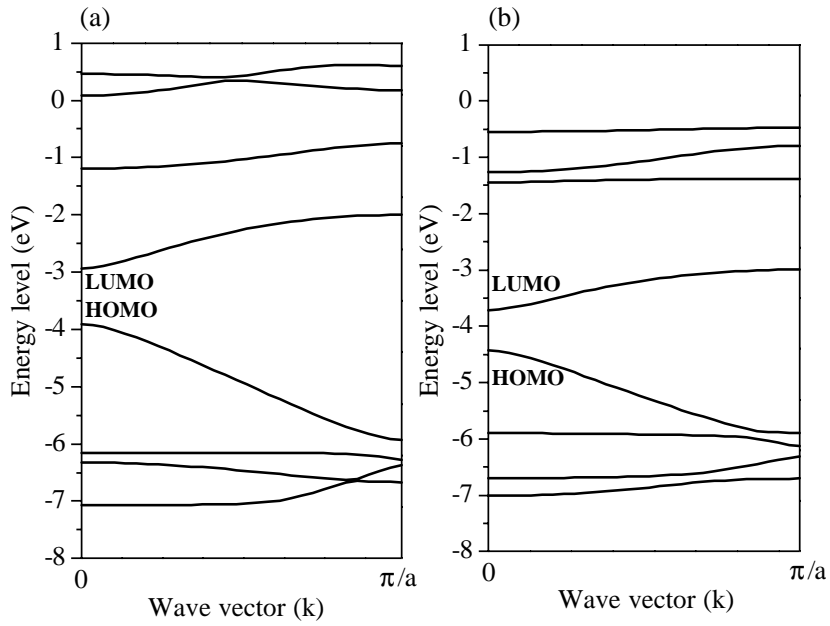

Fig. 7. Band structures of (a) PEDOT-TP and (b) PEDOT-BDP. $\pi$-conjugation was concluded from the geometrical analysis of these three polymers. The $m_{\mathrm{H}}$ of PEDOT-TP $\left(-0.104 m_{\mathrm{e}}\right)$ and PEDOT-BDP $\left(-0.110 m_{\mathrm{e}}\right)$ are smaller than that of polythiophene (PT) $\left(-0.133 m_{\mathrm{e}}\right)$ which suggests that these particular copolymers are good potential p-channel semiconductors for thin film transistors. The characteristic of the acceptor moieties also make these two copolymers possible $\mathrm{n}$-channel semiconductors for thin film transistors. The large bandwidths of these two copolymers also suggest extensive carrier delocalization and potential for good transport. Thus, PEDOT-TP and PEDOT-BDP could have potential applications as semiconductors for ambipolar organic field effect transistors.

\section{Conclusions}

The electronic structure and properties of 3,4-ethylenedioxythiophene (EDOT) based alternating donor-acceptor conjugated copolymers and their model compounds were systematically studied by DFT. The geometries of the materials were significantly affected by the acceptor ring size and intramolecular charge transfer. The HOMO level, LUMO level, and band gap of the model compounds were well controlled by the acceptor strength. However, the electronic properties of the copolymers did not exhibit a systematic trend with the acceptor strength due to a geometrical transformation going from the model compound to the polymer. PEDOT-TP and PEDOT-BDP have $E_{\mathrm{g}}$ smaller than $1.0 \mathrm{eV}$ due to their quinoid geometry and large intramolecular charge transfer. Hence, these two copolymers could have potential applications in transparent conductors, photovoltaic devices, or ambipolar thin film transistors. The substitution of nitrogen atoms on the backbone of PEDOT-Z, and PEDOT-D lowers the LUMO energy but results in the large band gaps due to electron localization. The $\pi$-conjugation disruption of PEDOT-TD, PEDOT-TPD, and PEDOT-BDD through the evolution of monomers to polymers contributes to their high band gaps in spite of apparently good intramolecular charge transfer. The theoretical results suggest that both the acceptor strength and the stable geometry contribute significantly to the electronic properties of alternating donor-acceptor conjugated copolymers. 


\section{Acknowledgements}

The work at National Taiwan University was supported by the National Science Council, the Ministry of Education, and the Ministry of Economic Affairs of Taiwan, ROC. That at University of Washington was supported by the Air Force Office of Scientific Research (Grant F49620-03-1-0162).

\section{Supplementary data}

Supplementary data associated with this article can be found at doi:10.1016/j.polymer.2005.11.083

\section{References}

[1] Roncali J. Chem Rev 1997;97:173.

[2] van Mullekom HAM, Vekemans JAJM, Havinga EE, Meijer EW. Mater Sci Eng B 2001;32:1.

[3] Ajayaghosh A. Chem Soc Rev 2003;32:181.

[4] Thompson BC, Madrigal LG, Pinto MR, Kang TS, Schanze KS, Reynolds JR. J Polym Sci, Part A: Polym Chem 2005;43:1417.

[5] Kulkarni AP, Zhu Y, Jenekhe SA. Macromolecules 2005;38:1553.

[6] Babel A, Wind JD, Jenekhe SA. Adv Funct Mater 2004;14:891.

[7] Champion RD, Cheng KF, Pai CL, Chen WC, Jenekhe SA. Macromol Rapid Commun 2005;26:1835.

[8] Yu G, Gao J, Hummelen JC, Wudl F, Heeger AJ. Science 1995;270:1789.

[9] Alam MM, Jenekhe SA. Chem Mater 2004;16:4647.

[10] Yamamoto T, Zhou ZH, Kanbara T, Shimura M, Kizu K, Maruyama T, et al. J Am Chem Soc 1996;118:10389.

[11] Tachibana M, Tanaka S, Yamashita Y, Yoshizawa K. J Phys Chem B 2002;106:3549.
[12] Yasuda T, Imase T, Nakamura Y, Yamatomo T. Macromolecules 2005; 38: 1500 .

[13] Thomas CA, Zong K, Abboud KA, Steel PJ, Reynolds JR. J Am Chem Soc 2004;126:16440.

[14] Groenendaal LB, Jonas F, Freitag D, Pielartzik H, Reynolds JR. Adv Mater 2000;12:481.

[15] Groenedaal LB, Zotti G, Aubert PH, Waybright SM, Reynolds JR. Adv Mater 2003;15:855.

[16] Zhu SS, Swager TM. J Am Chem Soc 1997;119:12568.

[17] Dubois CJ, Abboud KA, Reynolds JR. J Phys Chem B 2004;108: 8550 .

[18] Huang H, Pickup PG. Chem Mater 1998;10:2212.

[19] Berlin A, Zanelli A. Chem Mater 2004;16:3667.

[20] Akoudad S, Roncali J. Chem Commun 1998;9:2081.

[21] Perepichka IF, Levillain E, Roncali J. J Mater Chem 2004;14:1679.

[22] Raimundo JM, Blanchard P, Brisset H, Akoudad S, Roncali J. Chem Commun 2000;11:939.

[23] Meng H, Tucker D, Chaffins S, Chen Y, Helgeson R, Dunn B, et al. Adv Mater 2003;15:146.

[24] Frisch MJ, Trucks GW, Schlegel HB, Scuseria GE, Robb MA, Cheeseman JR, et al. Gaussian Inc.: Pittsburgh, PA; 2003.

[25] Delgado MCR, Hernandez V, Navarrete JTL, Tanaka S, Yamashita Y. J Phys Chem B 2004;108:2516.

[26] Satzner U. J Phys Chem B 2003;107:1129.

[27] Satzner U, Pickup PG, Poirier RA, Lagowski JB. J Phys Chem A 1998; 102:2572.

[28] Akoudad S, Roncali J. Synth Met 1999;101:149.

[29] Pepitone MF, Hardaker SS, Gregory RV. Chem Mater 2003;15:557.

[30] Brocks G, Havinga EE. Synth Met 2001;119:93.

[31] Bakhshi AK, Ago H, Yoshizawa K, Tanaka K, Yamabe T. J Chem Phys 1996; $104: 5528$.

[32] Chen WC, Jenekhe SA. Macromolecules 1995;28:465.

[33] Chen WC, Liu CL, Yen CT, Tsai FC, Tonzola CJ, Olson N, et al. Macromoelcues 2004;37:5959.

[34] Kürti J, Surján PR, Kertesz M. J Am Chem Soc 1991;113:9865. 